\title{
Frequency Parameters of the Myoelectric Signal as a Measure of Muscle Conduction Velocity
}

\author{
FOSTER B. STULEN, MEMBER, IEEE, AND CARLO J. DE LUCA, SENIOR MEMBER, IEEE
}

\begin{abstract}
During a sustained muscle contraction, the spectrum of the myoelectric signal is known to undergo compression as a function of time. Previous investigators have shown that the frequency compression is related to the decreasing conduction velocity of the muscle fibers. It is proposed that the frequency compression may be tracked by obtaining a continuous estimate of a characteristic frequency of the spectrum, such as the mean and median, or the ratio of low-frequency components to high-frequency components of the spectrum. A theoretical analysis was performed to investigate the restrictions in estimating the three parameters, as well as their sensitivity to the conduction velocity. The ratio parameter was found to be most sensitive to conduction velocity, but was the least reliable of the three. The median frequency was the least sensitive to noise. Therefore, from a theoretical point of view, the median frequency is the preferred parameter. A technique is described which determines an unbiased consistent estimate of the median frequency. The technique may be readily implemented in analog hardware.
\end{abstract}

\section{INTRODUCTION}

$\mathrm{I}$ N THE study of localized muscular fatigue, the analysis of the myoelectric (ME) signal has been employed extensively. Since the historic work of Piper [1], the frequencies of the ME signal have been known to decrease during a sustained contraction. Cobb and Forbes [2] noted this shift in the frequency components with fatigue, but also observed a consistent increase in amplitude of the ME signals recorded with surface electrodes. Many other investigators have noted an increase in ME signal amplitude [3]-[11]. The frequency shift has also often been observed [12]-[19].

Lindström et al. [15] have developed a mathematical model of the power density spectrum of the ME signal which explicitly depends on the conduction velocity of action potentials along the muscle fibers. Their model demonstrates that both the shift in the spectrum towards lower frequencies and the increase in the ME signal during a sustained contraction may be accounted by a single physiological correlate, the conduction velocity. If the model is indeed faithful to the underlying physiology, then either the changes in the frequency content or the amplitude of the ME signal ought to be useful for determining changes in the conduction velocity and, therefore, the progression of muscle fatigue.

Manuscript received March 24, 1980; revised March 4, 1981. This work was supported in part by the Rehabilitation Services Administration, U.S. Department of Health, Education, and Welfare under Grant 23-P-55854; by the Harvard-M.1.T. Program in Health Science and Technology; and by the National Institutes of Health under a Biomedical Research Grant to M.I.T.

F. B. Stulen was with the Department of Mechanical Engineering, Massachusetts Institute of Technology, Cambridge, MA 02139. He is now with Battelle Columbus Laboratories, Columbus, OH 43201.

C. J. De Luca is with the Neuromuscular Research Laboratory, Harvard Medical School, Children's Hospital Medical Center, Boston, MA 02115, and the Department of Mechanical Engineering, Massachusetts Institute of Technology, Cambridge, MA 02139.
Several investigators have in fact attempted to use the change in amplitude as an empirical measure of muscle fatigue $[6],[20],[8]$. Although the amplitude is indeed dependent on the conduction velocity, it is a second-order effect [21]. Furthermore, the amplitude is also known to vary with the type of electrode used to detect the signal, as well as the placement of the electrode. Stulen and De Luca [11] analyzed the amplitude change in 11 subjects performing sustained contractions at the identical relative force levels. Even when the amplitude was normalized, the variations could be as high as 30 percent. Thus, the use of a simple amplitude parameter of the ME signal is unsuitable for measuring the conduction velocity and the associated muscle fatigue.

The model of Lindström et al. [15] has shown that what has been referred to as a spectral shift is more accurately described as a spectral compression. Hence, to monitor the decrease in conduction velocity, it is only necessary to track the changes in a characteristic frequency of the spectrum. Some preferred candidates for the characteristic frequency are the mode, the mean, and the median frequency of the spectrum. In this article, the suitability of these three characteristic frequencies measuring the conduction velocity of the muscle fibers is examined. Another parameter associated with the median frequency, the ratio parameter, is also examined.

\section{MOdel For the Myoelectric Signal}

To examine the behavior of the spectral parameters, a model for the ME signal is required. The model should reasonably represent the ME signal which is detected with differential surface electrodes and must explicitly depend on the conduction velocity of the muscle fibers.

The surface ME signal is assumed to be a Gaussian random process. Several investigators have observed that the amplitude of ME signals obtained with surface electrodes is indeed well described by a Gaussian distribution [22]-[24]. Note that such a description for an ME signal composed of one or a very few motor unit action potential trains would be inappropriate since there are finite time intervals in a train when no motor unit action potential is present. Although the instantaneous amplitude of ME signals obtained with surface electrodes may be well described as a Gaussian random variable, it is not a sufficient condition for describing the ME signal as a Gaussian random process. Yet, for the purposes of this analysis, the ME signal is assumed to be a Gaussian random process because it greatly simplifies the analysis without compromising the results.

The assumption of a Gaussian random process significantly defines the ME signal; however, it does not define the shape of the spectrum of the signal. Therefore, it is assumed that the power density spectrum of the ME signal can bə expressed as a 


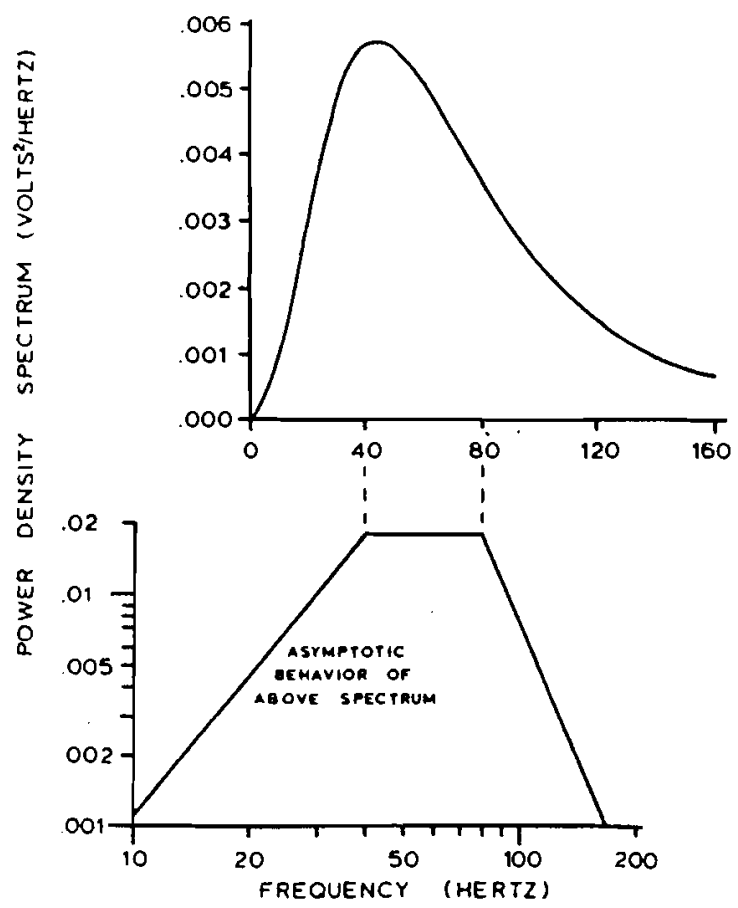

(a)

Fig. 1. Model for the power density spectrum of the myoelectric signal.

rational function of frequency. That is, the spectrum is assumed to be a ratio of two polynomials in frequency and, furthermore, that only even powers of frequency have nonzero coefficients. The latter assumption allows the signal to be modeled later as white noise passing through a linear filter. The order and coefficients of the polynomials are determined by empirically obtained power spectra of ME signals.

Hogan [25] and Shwedyk et al. [26] both observed that the power density spectra of surface ME signals obtained from the biceps brachii are accurately described by a rational function of frequency. Both observed a flat asymptotic behavior about the mode (peak) of the spectrum and the similar rolloff in spectrum as frequencies increased above this region. Below the flat region of the spectrum, Shwedyk et al. [26] noted a more shallow rolloff in the spectrum as frequency decreased than did Hogan [25]. The shallower rolloff is . lplies lower order polynomials which yield a simpler expression for the shape of the spectrum. Thus, a rational function of the form observed by Shwedyk et al. [26] is selected for the shape of the spectrum

$$
G(f)=\frac{k^{2} f_{h}^{4} f^{2}}{\left(f^{2}+f_{l}^{2}\right)\left(f^{2}+f_{h}^{2}\right)^{2}}
$$

where $G(f)$ denotes the shape of the spectrum as a function of frequency $f$ with $f_{l}$ and $f_{h}$ being the low and high cutoff frequencies, respectively, and $k^{2}$ being a scaling factor. Fig. 1(a) presents this spectrum plotted as a function of frequency and Fig. 1(b) presents the asymptotic behavior of this spectrum.

While this rational function approximation of the spectrum is adequate for the discussion presented here, there are two features which have been omitted. The first is the presence of "dips" in the high-frequency region of the spectrum. The occurrence of these "dips" were first predicted and observed by Lindström et al. [15]. More recently they have also been ob. served by Hogan [25] and others. The second is the occurrence of peaks in the low-frequency region of the spectrum. These peaks, which were predicted by LeFever and De Luca [27] and Lago and Jones [28], are dependent on the statistics of the firing patterns of the motor units. The analysis performed by LeFever and De Luca [27] showed that when many motor units are active, each with slightly different statistics, the peaks tend to average out. This condition is best met with surface electrodes for which the model is being developed.

In the model of Lindström et al. [15], the Fourier transform of the ME signal may be used to derive the following expres. sion for the spectrum:

$$
S(f)=\left(1 / v^{2}\right) G(f d / v)
$$

where $v$ is the conduction velocity. It can be seen from this expression that the conduction velocity explicitly affects signal amplitude by a factor of $1 / u$. The conduction velocity also inversely scales the frequency components of $G$, so that a decrease in conduction velocity compresses the spectrum. The frequency is also scaled by the factor $d$, which is the interelectrode distance of the bipolar-recording electrode. For convenience, $d$ is assumed to have a value of 1 . Conceivably, this scaling factor could be maintained throughout the following equations, but it would serve no useful purpose.

The previous equations may be combined resulting in the following expression:

$$
S(f)=\frac{\left(1 / v^{2}\right) k^{2} f_{h}^{4}(f / v)^{2}}{\left[(f / v)^{2}+\rho^{2} f_{h}^{2}\right]\left[(f / v)^{2}+f_{h}^{2}\right]^{2}}
$$

where $f_{l}$ has been expressed as a fraction of $f_{h}$,

$$
f_{l}=\rho f_{h} \quad \text { for } 0<\rho<1 .
$$

Although this mathematical expression is at best an approximation of an actual ME spectrum, it does explicitly depend on conduction velocity and represents a shape typical of ME sig. nals obtained with surface electrodes.

\section{Median and Mean Frequencies}

Since all the frequencies are scaled by the same factor, a spectral compression may be observed by tracking any characteristic frequency. Two obvious candidates are the median and mean frequencies. The median frequency $f_{\text {med }}$ is the frequency at which the spectrum is divided into two regions with equal power. This condition may be stated mathematically by the following equation:

$$
\int_{0}^{f_{\text {med }}} S(f) d f=\int_{f_{\text {med }}}^{\infty} S(f) d f=\frac{1}{2} \int_{0}^{\infty} S(f) d f .
$$

The mean frequency $f_{\text {mean }}$ is the average frequency and may be expressed by

$$
f_{\text {mean }}=\frac{\int_{0}^{\infty} f S(f) d f}{\int_{0}^{\infty} S(f) d f}
$$




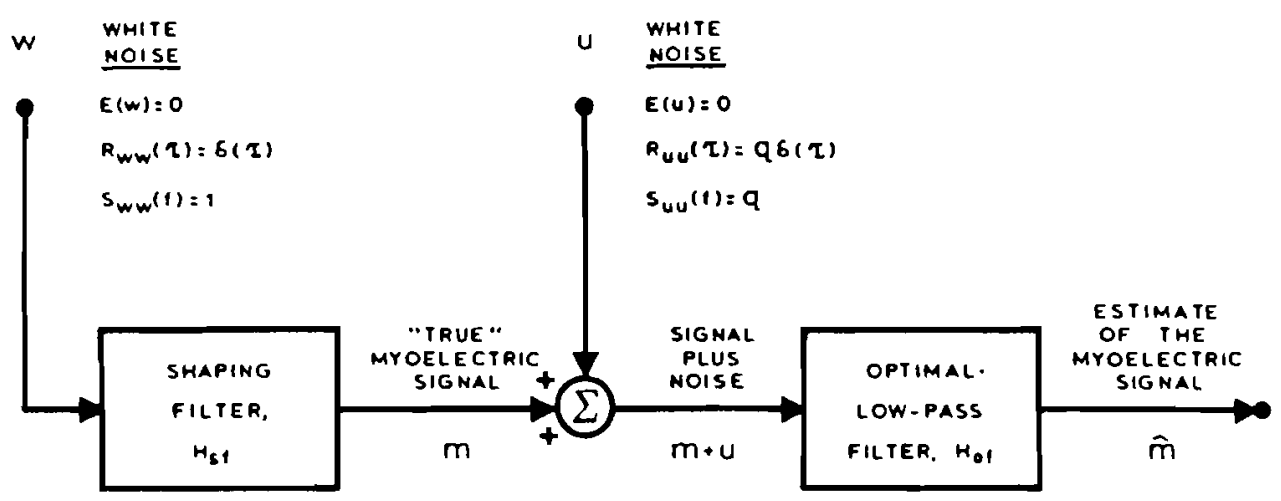

TRANSFER FUNCTIONS

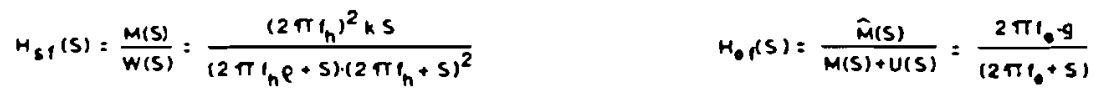

Fig. 2. Signal plus noise model of the myoelectric signal.

The signal obtained from a contracting muscle contains the desired ME signal $m$ and some ambient noise $u$ from the record. ing arrangement and equipment. The addition of this noise leads to errors in the subsequent estimation of the characteristic frequencies. The effects of noise are examined with the scheme shown in Fig. 2. The ME signal is generated by passing white noise through a shaping filter whose transfer function has the form

$$
H_{s f}(s)=\frac{k s\left(2 \pi f_{h}\right)^{2}}{\left(s+\rho 2 \pi f_{h}\right)\left(s+2 \pi f_{h}\right)^{2}}
$$

The power density spectrum of the white noise is $S_{w w}=1$. The power density spectrum of the output is the ME spectrum defined by (3). For this analysis, the conduction velocity is assumed to be constant and equal to one. This represents tine desired ME signal $m$. White noise $u$ is added to the desired signal and the result represents the observable ME signal. In reality, the noise is band limited; however, it may extend well beyond the frequency range of the desired signal. This situation may be modeled by passing the observed ME signal through a low-pass filter whose gain and cutoff frequency are optimally selected. Then, the output of this filter $\hat{m}$ becomes an estimate of the desired signal $m$. The gain and cutoff frequency are chosen to minimize the average steady-state squared error, $E\left[(m-\hat{m})^{2}\right]$. The derivation is presented in the Appendix.

Although Shwedyk et al. [26] did not state the break frequencies, they appear to be approximately 40 and $80 \mathrm{~Hz}$ from the data presented in their paper. Thus a $\rho$ of 0.5 was used for the analysis. The amplitude of noise was set at several different levels. For each noise level the optimal filter was determined. The median and mean frequencies of the output $\hat{m}$ of the optimal filter were determined. The percentage error between these frequencies and the median and mean frequencies of the desired signal $m$ were calculated. The signal-to-noise ratio (SNR) was calculated as the ratio of signal power in $\hat{m}$ due to the desired signal $m$ to the signal power in $\hat{m}$ due to the noise $u$.
The percentage error in the median and mean frequencies are plotted as a function of SNR in Fig. 3. The results show that the error in median frequency is less than the error in the mean frequency for any SNR. Even at relatively poor levels of SNR between 3 and 10, the error in median frequency is less than 10 percent, while for the mean frequency the error ranges between 55 and 20 percent. When the SNR is above 100 the error is negligible in both. This error is inherent to the signal acquisition and amplification system used. It represents a bias in the median and mean frequencies which is introduced before any technique is used to actually determine the frequencies. The final bias, accuracy, stability, and resolution of the estimates of the median and mean frequency are also strongly de. pendent on the technique and its implementation.

\section{The Ratio Parameter}

An alternative approach for tracking the spectral compression is by monitoring the ratio of the rms value of the highfrequency components to the rms value of the low-frequency components. The separation point between the high- and low-frequency regions may be any convenient characteristic frequency, such as the mean or median of the spectrum, chosen at the initiation of a contraction. This parameter is very appealing and convenient to monitor and, in fact, has been used by some investigators. However, this parameter has several drawbacks because of its dependence on the spectral shape and the value of the initial characteristic frequency. These points become evident by the following analysis.

The ratio parameter nay be mathematically expressed by

$$
R=\left[\frac{\int_{0}^{f_{\mathrm{chr}}^{0}} S(f / v) d f}{\int_{f \mathrm{chr}}^{\infty} S(f / v) d f}\right]^{1 / 2}
$$

where $R$ is the ratio parameter and $f_{\text {chr }}^{0}$ is the initial character- 


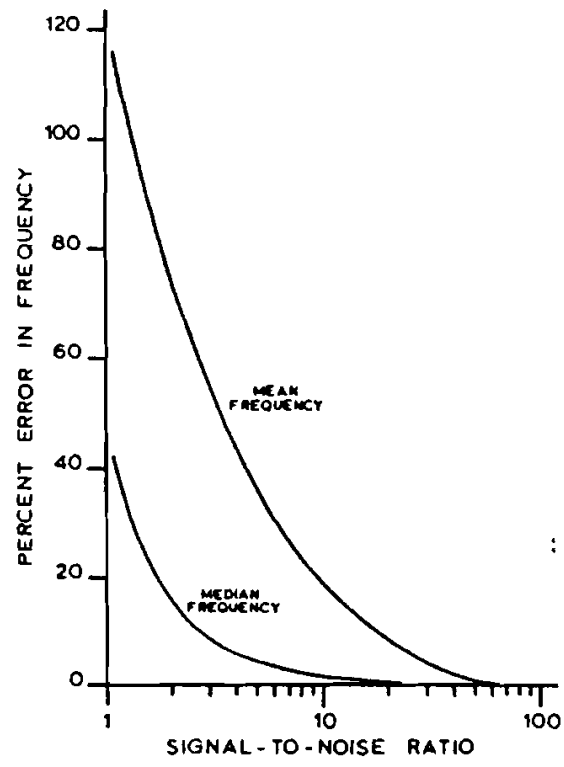

Fig. 3. Percentage error in the mean and median frequency of the myoelectric signal as a function of signal-to-noise ratio.

istic frequency. For the purpose of this analysis the median frequency was used as the characteristic frequency. The above expression for the ratio parameter may be evaluated by substituting (3) into (7) and integrating by parts. This expression was evaluated for several spectra of the form given by (3) with $\rho=0.1,0.3,0.5,0.7$ as a function of conduction velocity normalized by its initial value. The results are shown in Fig. 4. The plots indicate that the ratio parameter is sensitive to the shape of the spectrum as indicated by the family of curves generated for $\rho$. This is a drawback since in practice the spectral shape of the ME signal is not exactly known and, furthermore, is variable among muscles, and among subjects [29].

Another detraction of this parameter is its dependence on the determination of the initial characteristic frequency. In practice the value of the characteristic frequency derived from any technique is an estimate of the true value. Hence, the ratio represents one sample curve for a sample value of the initial characteristic frequency. A Monte Carlo simulation was used to determine the bias and variance in the ratio parameter for a given coefficient of variation in the initial characteristic frequency.

The distribution of the initial median frequency was assumed to be Gaussian with a mean which equalled the "true" initial median frequency $f_{\text {chr }}$ and variance $\sigma^{2}$. A total of 200 sample values of the initial median frequency was determined and the corresponding ratio curve was obtained as a function of normalized conduction velocity $V$. The percentage bias in the ratio was estimated by

$$
\frac{\frac{1}{N} \sum_{i=1}^{N} R_{i}(V)-R^{*}(V)}{R^{*}(V)}
$$

where $R_{i}(V)$ is the ratio curve for a given sample value of initial median frequency, $R^{*}(V)$ is the ratio curve for the true initial median frequency, and $N$ equals 200 . The coefficient of variation in the ratio as a function of conduction velocity was

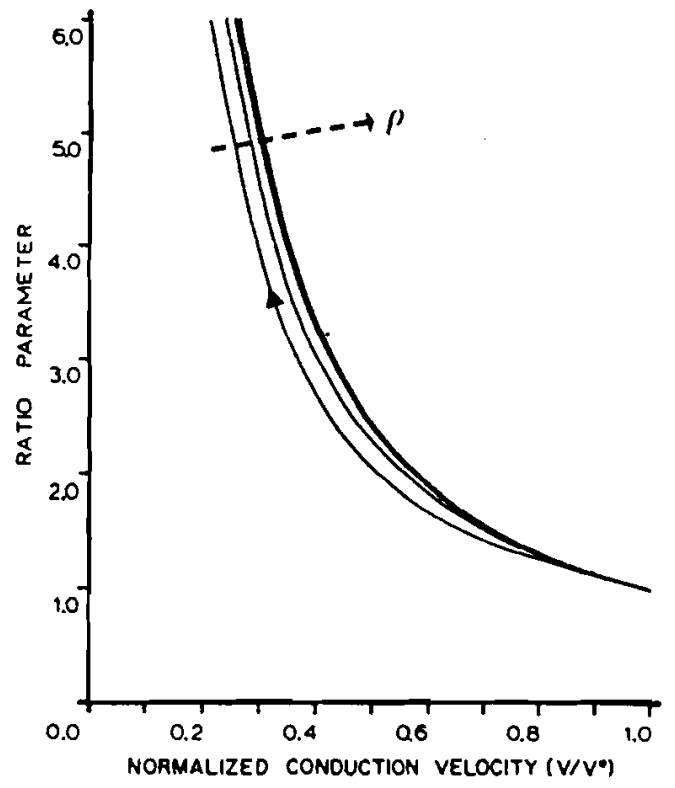

Fig. 4. Ratio parameter as a function of the change in the conduction velocity of the muscle fibers. The conduction velocity was normalized to the value at the beginning of the muscle contraction $\left(v^{0}\right)$, and it decreases as contraction time increases. The four curves are obtained by using slightly different shapes of the myoelectric signal spectrum as indicated by the parameter $\rho(0.1,0.3,0.5,0.7)$. See text for details.

also obtained. (The coefficient of variation is the ratio of standard deviation to the mean.) The percentage bias and coefficient of variation of the ratio parameter for a spectrum of the form of (3) with $\rho=0.5$ are plotted in Fig. 5 for different coefficients of variations in the initial median frequency. There are two major observations that may be made in Fig. 5. First, there is a nonzero bias in the ratio parameter which is dependent on the coefficient of variation of the initial median frequency. Second, the coefficient of variation in the ratio parameter is approximately 30 percent greater than that in the initial median frequency.

\section{Sensitivity to Conduction Velocity}

Since the parameters of the spectrum are all dependent on the conduction velocity, it is necessary to determine the degree of their dependence (sensitivity) on the conduction velocity. For the median frequency the necessary expression may be obtained by substituting (2) in'o (4):

$$
\int_{0}^{f_{\text {med }}} \frac{G(f / v)}{v^{2}} d f=\frac{1}{2} \int_{0}^{\infty} \frac{G(f / v)}{v^{2}} d f
$$

which reduces to

$$
\int_{0}^{f_{\mathrm{med}} / v} G(f / v) d(f / v)=\frac{1}{2} \int_{0}^{\infty} G(f / v) d(f / v)
$$

The right-hand side of the above equation is constant, hence the upper limit of integration of the left-hand integral must be constant. If one defines the initial medial frequency $f_{\text {med }}^{0}$ as the median frequency when the conduction elocity is at its initial value $v^{0}$ then 


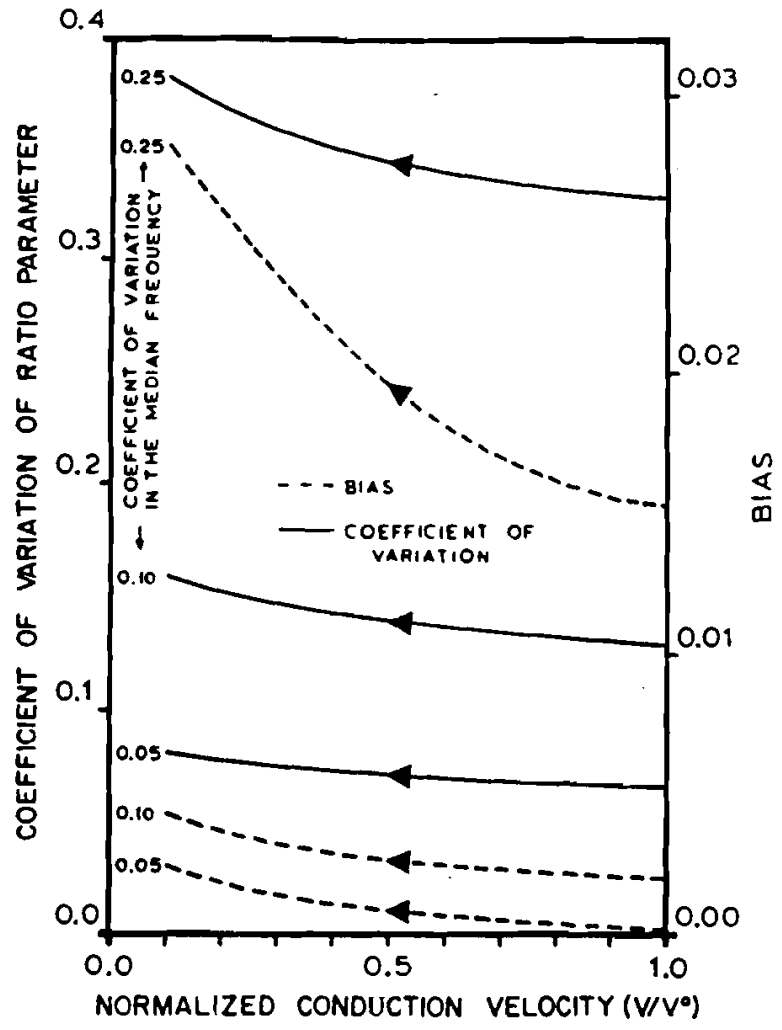

Fig. 5. Coefficient of variation and bias of the ratio parameter as a function of the conduction velocity of the muscle fibers. The curves were obtained for three values of the coefficient of variation of the initial median frequency. The conduction velocity was normalized to the value at the beginning of the muscle contraction $\left(v^{0}\right)$, and it decreases as contraction time increases.

$$
f_{\text {med }}=\left(\frac{v}{v^{0}}\right) f_{\text {med }}^{0}
$$

The median frequency is therefore linearly proportional to the conduction velocity.

The expression describing the sensitivity of the mean frequency to the conduction velocity may be obtained by substituting (2) into (5):

$$
f_{\text {mean }}=v \cdot \frac{\int_{0}^{\infty}(f / v) G(f / v) d(f / v)}{\int_{0}^{\infty} G(f / v) d(f / v)} .
$$

In this case, the quotient of integrals is a constant $f_{\text {mean }} / v$ hence

$$
f_{\text {mean }}=\left(\frac{v}{v^{0}}\right) f_{\text {mean }}^{0}
$$

The expressions [(11) and (13)] for both characteristic frequencies are equivalent. In fact, any characteristic frequency will be linearly proportional to the conduction velocity, since all frequencies of the ME signal are scaled inversely by the conduction velocity. However, other parameters that are associated with the spectrum are not necessarily linearly dependent on the conduction velocity. Such is the case for the ratio parameter. The necessary expression is given by (7).

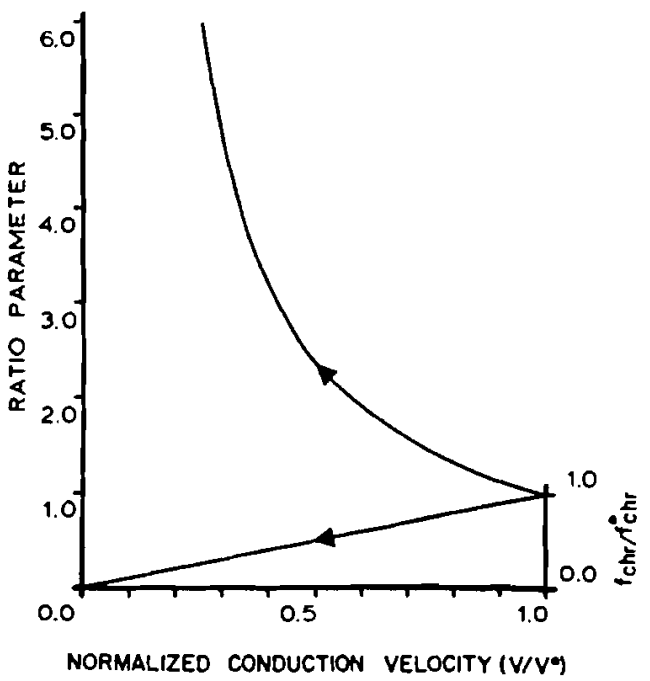

Fig. 6. Relationship between the ratio parameter and characteristic frequencies and the conduction velocity of the muscle fibers. The conduction velocity was normalized to the value at the beginning of the muscle contraction $\left(v^{0}\right)$, and it decreases as contraction time increases.

Fig. 6 presents a plot of the relationships of the characteristic frequencies and ratio parameter as a function of conduction velocity. These are plots of (7) and (11). The value of the characteristic frequency and conduction velocity have been normalized to one, which represents the value at the beginning of a muscle contraction when no significant metabolic changes have occurred in the muscle. The characteristic frequencies decrease linearly and the ratio parameter increases nonlinearly as the conduction velocity decreases, as would be the case during a sustained contraction. The value of the characteristic frequency progresses towards zero, whereas the value of the ratio parameter approaches infinity as the value of the conduction velocity approaches zero.

It should be noted that although in Figs. 5 and 6 the spectral parameters have been calculated for conduction velocity values approaching zero, this situation does not occur in healthy muscles. In fact, the greatest decrease in the median frequency observed by Stulen [30] was to 30 percent of the initial value during a sustained isometric constant-force contraction of the first dorsal interosseous muscle.

\section{A Technique to Estimate the Median Frequency}

Of the three parameters considered, it appears that under the conditions of the performed analyses, the median frequency provides the most accurate estimate of the conduction velocity. Therefore, a technique was developed for determining the median frequency of an ME signal on line and in real time. A schematic of the technique is shown in Fig. 7. The ME signal is passed through modulated low-pass and high-pass filters whose cutoff frequencies are constrained to be equal, but can be varied by a control voltage. The outputs of the filters are connected to nonlinear devices whose outputs are estimates of their variances (or monotonic function of the variance) of the input. These are in turn connected to a difference amplifier which is connected to an integrator. The output of the integrator varies the cutoff frequency of the filters. Thus, when the variances of the two outputs of the filters are equal, the 


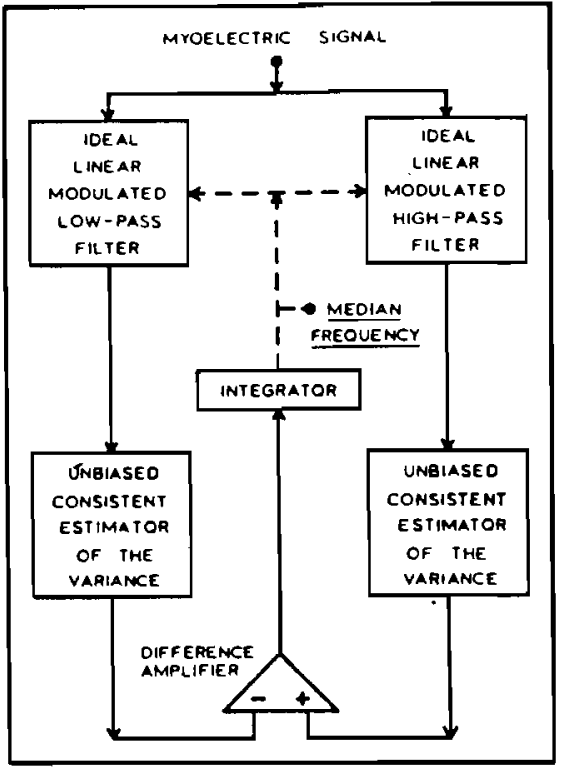

Fig. 7. Technique for estimating the median frequency of the myoelectric signal.

difference $\Delta$ is zero and the mean value of the integrator output corresponds to the median frequency. When the integrator output is not at the median frequency there will be a difference in the variance of the two signals. This difference forces the integrator output to vary about the value that corresponds to the median frequency.

The performance of the proposed technique may be analyzed in the following manner. The input to the low-pass and high-pass filters is assumed to be a Gaussian random process with zero mean. The filters are linear, which implies that the output is also a Gaussian random process. The unbiased consistent estimator of the signal variance may be synthesized by a squaring circuit cascaded with a linear integral averager. Under these conditions the output of the averager provides an unbiased estimate of the variance of the filtered signal. Furthermore, if the integral of the squared correlation function of the input is finite, then the estimate is also consistent. These operations form the left and right pathways in the technique for the estimation of the median frequency shown in Fig. 7.

The mean value of the difference operation is the difference of the variances of the outputs of the low-pass and high-pass filters. The variance of the difference is dependent on the correlation of the output of the averages, and may be expressed as

$$
\begin{aligned}
\operatorname{var}(\Delta) & =E\left\{[\Delta-E(\Delta)]^{2}\right\} \\
& =\operatorname{var} \hat{\sigma}_{h}^{2}+\operatorname{var} \hat{\sigma}_{l}^{2}-2\left[E\left(\hat{\sigma}_{l}^{2} \hat{\sigma}_{h}^{2}\right)-\sigma_{l}^{2} \sigma_{h}^{2}\right]
\end{aligned}
$$

where $\Delta$ is the difference, $\sigma^{2}$ is the variance, $\hat{\sigma}^{2}$ is the estimate of the variance, and the subscripts $l, h$ denote low-pass and high-pass signals. When the outputs of the filters are uncorrelated (linearly independent), (14) reduces to

$$
\operatorname{var}(\Delta)=\operatorname{var}\left(\hat{\sigma}_{l}^{2}\right)+\operatorname{var}\left(\hat{\sigma}_{h}^{2}\right) \text {. }
$$

This relationship is valid for ideal high-pass and low-pass filters with frequency responses

$$
\begin{array}{rlrl}
H_{h}(f) & =1 & & |f|>f_{c} \\
& =0 & & |f|<f_{c} \\
H_{l}(f)=1 & & |f|<f_{c} \\
& =0 & & |f|>f_{c}
\end{array}
$$

because they occupy disjoint regions of frequency [31]. The term $f_{c}$ denotes the cutoff frequency of the filters.

When the cutoff frequency of the filters is set at the median frequency, then the average of the difference is zero. In practice, the cutoff frequency is not set, but is varied by the integral of the difference, thus forming a feedback system. If this system is stable, the cutoff frequency is driven to the median frequency. Once settled about the median frequency, the average value of the difference is zero and the mean of the output of the integrator corresponds to the median frequency. Although the cutoff frequency would tend to drift away from the median frequency because of the integration of a random process, the feedback nature of the system corrects any errors. As a result, the variance of the output of the integrator is finite. The rate at which the system responds must be slower than the lowest frequency in the signal. Otherwise, the dynamics of the feedback system will have a significant effect on the two filtered signals.

The dynamic performance of the technique is dependent on the statistical properties of the input signal. The above analysis has assumed that the input signal is stationary. In actuality, the statistical properties of the ME signal are nonstationary during a sustained contraction. One of the main advantages of the technique is that it allows continuous on-line monitoring of the nonstationarity of the signal by observing changes in the estimate of the median frequency. The nonstationarity creates a tradeoff between resolution and accuracy.

An analog implementation of the technique, known as the Muscle Fatigue Monitor (MFM) has been realized [32], [33]. In that implementation an analysis of the mean values of the variables is used to describe the system response. The analysis is then used to optimize the response of the system to a step change in the median frequency.

\section{Discussion}

All the frequency parameters discussed in this article have been used by several investigators to measure the compression of the ME signal spectrum during sustained muscle contractions. The mean frequency has been used by Hagberg [34], Herberts et al. [29], Broman and Kadafors [35], Ortengren et al. [36], Lynne-Davies [37] and others; the median frequency has been used by Stulen and De Luca [11], [32], Sabbahi et al. [38], and Petrofsky and Lind [39]. The ratio parameter has been used by Bellemare and Grassino [40], Gross et al. [41], [42], Kadefors et al. [14], Muller et al. [43], and Schweitzer et al. [44].

There is one additional parameter, the mode (peak) frequency of the spectrum, which deserves mention. The estimate of this parameter was not considered in the analysis because even for relatively poor signal-to-noise ratio (SNR), it is always theoretically possible to ol tain a perfect estimate. Therefore, superficially it would appear to be the most appropriate char- 
acteristic frequency. However, this is not the case because the ME signal is a stochastic signal which does not have a smooth and sharply defined region near the peak value of its spectrum. Furthermore, the variance of the spectrum would strongly influence the estimation accuracy of the mode. This point has been confirmed empirically by Schweitzer et al. [44]. They found that the coefficient of variation for the estimate of the mode was five times greater than that of the mean frequency for ME signals obtained from the human diaphragm.

The theoretical analysis indicates that the estimates of both the mean and median frequencies are affected negligibly by noise when the SNR is greater than 100 . In a more modest range of SNR, the error is far less in the median frequency than in the mean frequency. Even when the SNR is about 2, the error in the median frequency is less than 20 percent. These results were obtained using an optimal filter to minimize the effects of the noise. The filter directly depended on the spectrum of the signal. Hence, if the filter were optimal at the start of a sustained contraction, then as the spectral compression progresses, the errors in the characteristic frequencies would grow. The error increases much more in the mean frequency than in the median frequency. This problem could be counteracted by designing the filter so that the bandwidth is adjusted by the estimate of the characteristic frequency, thereby keeping the filter near optimal as the spectrum compresses. In fact, a device developed by Broman and Kadefors [35] does use multiple low-pass filters to minimize the effects of noise as it estimates the value of the mean frequency. However, because the median frequency is less sensitive to noise, only a single constant low-pass filter is necessary to obtain a similar improvement.

The ratio parameter as proposed by De Luca and Berenberg [45] has been shown to be sensitive to the shape of the ME signal frequency spectrum. This is a hindrance because in some muscles the frequency components of the ME signal may vary during varying-force contractions due to the recruitment of motor units that have significantly different action potential shapes. Furthermore, this parameter is dependent on the initial value of the characteristic frequency chosen to divide the spectrum. In practice, the true initial value of the characteristic frequency is not known and must be estimated. As a result, the observed ratio parameter is itself only an estimate of the true ratio value. For the case where the median frequency was used as the partitioning frequency, a statistical analysis has shown that the ratio parameter estimate is biased and has a covariance approximately 30 percent greater than the covariance of the estimate of the median frequency.

As may be seen in Fig. 6, the ratio parameter is the most sensitive to changes in the conduction velocity of the muscle fibers, but the relationship is nonlinear; whereas, the mean and median frequencies have an equal sensitivity to the conduction velocity. Although it is less than that of the ratio parameter, the sensitivity is linear.

In an empirical evaluation of the ratio parameter, the mode frequency, and the mean frequency, Schweitzer et al. [44] also concluded that the mean frequency was the most stable and reliable. From the theoretical basis discussed in this paper, the median frequency is in general a more superior estimator than the mean frequency for monitoring the changes in the conduction velocity of muscle fibers during a contraction. Therefore, the median frequency is the preferred parameter. However, it should be noted that the accuracy of the estimates of the considered parameters calculated from empirical data will also depend on the specifics of the actual techniques used to obtain the estimates. Hence, it is conceivable that in practice the mean frequency may provide an acceptable estimate of the conduction velocity.

\section{APPENDIX}

A signal is often low-pass filtered to reduce the contamination from ambient noise. In practice, the cut off frequency of the filter is chosen to be sufficiently high to pass the majority of the desired signal and sufficiently low to remove most of the higher frequency noise components. However, both the cutoff frequency and the gain of the filter may be determined optimally by minimizing the squared error between the output of the filter and the desired signal. This is the same principle as in Kalman filtering.

For the analysis, the propagation of the covariance of a random input through a linear system must be determined. Consider a linear system of first-order differential equations

$$
\dot{x}=A x+B y \text {. }
$$

The state of the system is defined by the column vector $x$, and is driven by the column vector of inputs $y$. When $y$ is composed of random inputs, the state of the system $x$ is also random and the covariance $K_{x x}(t, \tau)$ of the state is defined by

$$
K_{x x}(t, \tau)=E\left\{[x(t)-E[x(t)]] \cdot[x(\tau)-E[x(\tau)]]^{T}\right\}
$$

For convenience, when $\tau=t$, the covariance matrix will be denoted by

$$
P_{x}(t)=K_{x x}(t, t) \text {. }
$$

By the application of the theories of linear systems and probability, it can be shown that

$$
\frac{d}{d t} P_{x}(t)=A P_{x}(t)+P_{x}(t) A^{T}+B Q(t) B^{T}
$$

where

$$
Q(t) \cdot \delta(t-\tau)=E\left[y(t) \cdot y^{T}(\tau)\right]
$$

For the total solution of the above equation, the initial condition for $P_{x}(t)$ must be known. In steady state, (A4) reduces to

$$
A P_{x}^{s}+P_{x}^{s} A^{T}+B Q B^{T}=0
$$

where the superscript $s$ indicates steady state.

With the above results as background, the determination of an optimal filter can now be approached. The overall problem is depicted in Fig. 2. The myoelectric signal $m$ may be modeled as the output of a linear shaping filter whose input is Gaussian white noise. The transfer function of the filter is assumed to be

$$
H_{s f}(s)=\frac{M(s)}{W(s)}=\frac{k s\left(2 \pi f_{h}\right)^{2}}{\left[\rho 2 \pi f_{h}+s\right]\left[2 \pi f_{h}+s\right]^{2}}
$$


where $s$ is the Laplace operator and $M(s)$ and $W(s)$ are the Laplace transforms of the signal and white noise, respectively. The white noise is assumed to have a spectral amplitude of 1.0 and the gain of the filter $k$ is chosen so that the power of the resulting signal is arbitrarily set at 1.0 . White noise $u$ with spectral height $q$ is added to this desired signal. The combined signal is then passed through a single pole low-pass filter to remove the noise. The gain of the filter is denoted by $g$ and the cutoff frequency is denoted by $f_{0}$. The output of the second filter $\hat{m}$ is considered to be an estimate of the myoelectric signal $m$. The gain and cutoff frequency of the filter are to be chosen to minimize the average squared error between $m$ and $\hat{m}$. This system may be expressed in the form of (A1) as a set of four first-order linear differential equations forced by two random uncorrelated inputs $w$ and $u$ :

$$
\begin{aligned}
\frac{d}{d t}\left[\begin{array}{l}
m \\
m_{2} \\
m_{3} \\
\hat{m}
\end{array}\right]= & {\left[\begin{array}{cccc}
0 & 1 & 0 & 0 \\
0 & 0 & 1 & 0 \\
-\rho Z^{3} & -(1+2 \rho) Z^{2} & -(2+\rho) Z & 0 \\
g & 0 & 0 & f_{0}
\end{array}\right] } \\
& \cdot\left[\begin{array}{l}
m \\
m_{2} \\
m_{3} \\
\hat{m}
\end{array}\right]+\left[\begin{array}{cc}
0 & 0 \\
k Z^{2} & 0 \\
-(2+\rho) Z^{3} & 0 \\
0 & g
\end{array}\right]\left[\begin{array}{l}
w \\
u
\end{array}\right]
\end{aligned}
$$

With this formulation, the error may be expressed as

$$
E\left[(m-\hat{m})^{2}\right]=\left[\begin{array}{r}
1 \\
0 \\
0 \\
-1
\end{array}\right]^{T}\left[P_{x}^{s}\right]\left[\begin{array}{r}
1 \\
0 \\
0 \\
-1
\end{array}\right]=P_{11}-2 P_{14}+P_{44}
$$

where $P_{l j}$ is the element in the $i$ th row and the $j$ th column of $P_{x}^{s}$. In order to determine these elements, the covariance matrix of the inputs $Q$ is also needed.

$$
Q=\left[\begin{array}{ll}
1 & 0 \\
0 & q
\end{array}\right]
$$

The matrices $A, B$, and $Q$ are substituted into (A6) to determine the elements of $P_{x}^{s}$. The steady-state error is then determined by (A9). For a given spectrum and noise level, the error is a function of the gain and cutoff frequency of the filter. Thus, the optimal gain and cutoff frequency are those values which minimize the average squared error. An optimization routine was used to find the optimal gains and cutoff frequencies for several levels of spectral noise for the results presented in the text.

\section{ACKNOWLEDGMENT}

The authors wish to thank Dr. R. S. LeFever for his valued assistance in several details of the analysis and Prof. R.W.Mann for his advice throughout the project.

\section{REFERENCES}

[1] H. Piper, Electrophysiologie Muschliche Muskeln. Berlin, Germany: Springer, 1912, p. 126.

[2] S. Cobb and A. Forbes, "Electromyographic studies of muscle fatigue in man," Amer. J. Physiol., vol. 65, pp. $234-251,1923$.

[3] G. C. Knowlton, R. L. Bennett, and R. McClure, "Electromyography of fatigue," Arch. Phys. Med., vol. 32, pp. 648-652, 1951.

[4] O.C.J. Lippold, J.W.T. Redfeam, and J. Vućo, "The electromyography of fatigue," Ergonomics, vol. 3, pp. 121-131, 1970.

[5] R. G. Eason, "Electromyographic study of local and generalized muscular impairment," J. Appl. Physiol., vol. 15, pp. 479-482, 1960.

[6] H. A. DeVires, "Efficiency of electrical activity as a physiological measure of the functional state of muscle tissue," Amer. J. Phys. Med., vol. 47, pp. 10-22, 1968.

[7] E. Kuroda, V. Klissouras, and J. H. Milsum, "Electrical and metabolic activities and fatigue in human isometric contractions," $J$. Appl. Physiol., vol. 29, pp. 359-367, 1970.

[8] A. J. Lloyd, "Surface electromyography during sustained isometric contractions," J. Appl. Physiol., vol. 30, pp. 713-719, 1971.

[9] J. Vredenbregt and G. Rau, "Surface electromyography in relation to force, muscle length, and endurance," in New Developments in EMG and Clinical Neurophysiology, J. E. Desmedt, Ed. Basel, Switzerland: Karger, 1973, 607-622.

[10] J. A. Stephens and T. P. Usherwood, "The fatigability of human motor units," J. Physiol., vol. 250, pp. 37-38, 1975.

[11] F. B. Stulen and C. J. De Luca, "Median frequency of the myoelectric signal as a measure of localized muscular fatigue," in Proc. 4th Cong. Int. Soc. Electrophysiology and Kinesiology, Aug. 1979, pp. 92-93.

[12] K. Kogi and T. Hakamada, "Frequency analysis of the surface electromyogram in muscle fatigue," J. Sci. Labour (Tokyo), vol. 38, pp. 519-528, 1962.

[13] M. Sato, "Some problems in the quantative evaluation of muscle fatigue by frequency analysis of the electromyogram," J. Anthro. Soc. Japan, vol. 73, pp. 20-27, 1965.

[14] R. Kadefors, E. Kaiser, and I. Petersén, "Dynamic spectrum analysis of myo-potentials with special reference to muscle fatigue," Electromyography, vol. 8, pp. 39-74, 1968.

[15] L. Lindstrom, R. Magnusson, and I. Petersén, "Muscular fatigue and action potential conduction velocity changes studied with frequency analysis of EMG signals," Electromyography, vol. 10, pp. 341-356, 1970.

[16] D. B. Chaffin, "Localized muscle fatigue-Definition and mezsurement," J. Occupation. Med., vol. 15, pp. 346-354, 1973.

[17] A. Magora, B. Gonen, D. Eimeri, and F. Magora, "Electrophysiological manifestations of isometric contraction sustained to maximal fatigue in healthy humans," Electromyogr. clin. Neuro. physiol., vol. 16, pp. 309-334, 1976.

[18] J.H.T. Viitasalo and P. V. Komi, "Signal characteristics of EMG during fatigue," Europ. J. Appl. Physiol., vol. 37, pp. 111-121, 1977.

[19] M. W. Givens and J. B. Teeple, "Myoelectric frequency changes in children during static force production," Electroencephalogr. clin. Neurophysiol., vol. 45, pp. 173-177, 1978.

[20] D. P. Currier, "Measurement of muscle fatigue," Phys. Ther., vol. 49, pp. 724-730, 1969.

[21] L. Lindström, R. Kadefors, and I. Petersén, "An electromyographic index for localized muscle fatigue," J. Appl. Physiol.: Respirat., Environ., Exercise Physiol., vol. 43, pp. 750-754, 1977.

[22] E. Kaiser, R. Kadefors, R. Magnusson, and J. Petersén, "Myoelectric signals for prosthesis control," Medicinsk Teknik/Medico Teknik, vol. 1, pp. 14-42, 1968.

[23] E. Kwatny, D. H. Thomas, and H. G. Kwatny, "An application of signal processing techniques to the study of myoelectric signals," IEEE Trans. Biomed. Eng., vol. BME-17, pp. 303-313, 1970.

[24] H. Roesler, "Statistical analysis and evaluation of myoelectric signals for proportional control," in The Control of Upper-Extremity Prostheses and Orthosis, P. Herberts, R. Magnusson, R. Kadefors, and I. Petersen, Eds. Springfield, IL: Charles C Thomas, 1974.

[25] N. J. Hogan, "Myoelectric prosthesis control: Optimal estimation applied to EMG and the cybernetic considerations for its use in a man-machine interface," Ph.D. dissertation, Massachusetts Inst. Techn .l., Cambridge, MA, Aug. 1976. 
[26] E. Shwedyk, R. Balasubramanian, and R. N. Scott, "A nonstationary model for the electromyogram," IEEE rrans. Biomed. Eng., vol. BME-24, pp. 417-424,1977.

[27] R. S. LeFever and C. J. De Luca, "The contribution of individual motor units to the EMG power spectrum," in Proc. 29th Annu. Conf. Engineering in Medicine and Biology, 1976, p. 56.

[28] P. Lago and N. B. Jones, "Effect of motor-unit firing statistics on EMG spectra," Med. Biol. Eng. Comput., vol. 15, pp. 648-655, 1977.

[29] P. Herberts, E. Kaiser, R. Magnusson, and I. Petersén, "Power spectra of myoelectric signals in muscles of arm amputees and healthy normal controls," Acte Orthoped. Scand., vol. 39, pp. 132,1969 .

[30] F. B. Stulen, "A technique to monitor localized muscular fatigue using frequency domain analy sis for the myoelectric signal," Ph.D. dissertation, Massachusetts Inst. Technol., Cambridge, MA, 1980.

[31] A. Papoulis, Probability, Random Variables, and Stochastic Processes. New York: McGraw-Hill, 1965, p. 353.

[32] F. B. Stulen and C. J. De Luca, "A noninvasive device for monitoring metabolic correlates in myoelectric signals," in Proc. 31st Annu Conf. Engineering in Medicine and Biology, Oct. 1978, p. 264.

[33] - "A non-invasive device for monitoring localized muscular fatigue," in Proc. 14th Annu. Meeting Ass. Adrancement in Medical Instrumentation, May 1979, p. 268.

[34] M. Hagberg, "The elevated arm: myoelectric amplitude and spectral changes in some shoulder muscles," in Proc. 4th Cong. Int. Soc. Electrophysiology and Kinesiology, Aug. 1979, pp. 70-71.

[35] H. Broman and R. Kadefors, "A spectral moment analyzer for quantification of electromyograms," in Proc. 4th Cong. Int. Soc. Electrophysiology and Kinesiology, Aug. 1979, pp. 90-91.

[36] R. Örtengren, L. Lindström, and I. Petersén, "Electromyographic evaluation and modelling of localized fatigue during periodic muscular work," in Proc. 4th Cong. Int. Soc. Electrophysiology and Kinesiology, Aug. 1979, pp. 96-97.

[37] P. Lynne-Davies, M. Coker, D. Simkins, and B. Widrow, "Adaptive filtering in respirator electromyogram processing," in Proc. 4th Cong. Int. Soc. Electrophysiology and Kinesiology, 1979, pp. $170-171$.

[38] M. A. Sabbahi, C. J. De Luca, and W. R. Powers, "The effect of ischemia, cooling and local anesthesia on the median frequency of the myoelectric signal," in Proc. 4th Cong. Int. Soc. Electrophysiology and Kinesiology, Aug. 1979, pp. 94-95.

[39] J. S. Petrofsky and A. R. Lind, "Isometric strength, endurance, and the blood pressure and heart rate responses during isometric exercise in healthy men and women with special reference to age and body fat content," Pflugers Arch., vol. 360, pp. 49-61,1975.

[40] F. Bellemare and A. Grassino, "The fatigue and recovery of the human diaphragm," in Proc. 4th Cong. Int. Soc. Electrophysiology and Kinesiology, Aug. 1979, pp. 74-75.

[41] D. Gross, A. Grassino, W.R.D. Ross and P. T. Macklem, "Electromyogram pattern of diaphragmatic fatigue," J. Appl. Physiol.: Respirat., Environ., Exercise Physiol., vol. 46, pp. 1-7, 1979.

[42] D. Gross, A. Grassino, G. Scott, H. Ladd, and P. T. Macklem, "Respiratory muscle fatigue in quadriplegic patients," Clin. Res. vol. 25, p. $713 \mathrm{a}, 1978$

[43] N. Muller, G. Gulston, C. Cade. J. Whittan, M. H. Bryon, and A. C. Bryan, "Respiratory muscle fatigue in infants," Clin. Res. vol. 25 , p. $174 a, 1978$.
[44] T. W. Schweitzer, J. W. Fitzgerald, J. A. Bowden, and P. LynneDavies, "Spectral analysis of human inspiratory diaphragmatic electromyograms," J. Appl. Physiol.: Respirat., Environ., Exer. cise Physiol., vol. 46, pp. 152-165, 1979.

[45] C. J. De Luca and W. Berenberg, "A polar technique for displaying EMG signals," in Proc. 28th Annu. Conf. Engineering in Med. cine and Biology, 1975, p. 21.

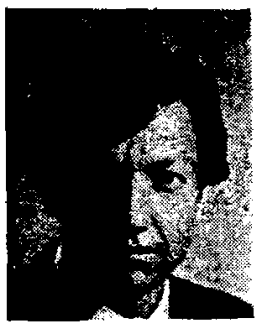

Foster B. Stulen (S'78-M'79) was born in Morristown, NJ, on November 3, 1951. He received the B.S. degree in mechanical engineering in 1973 from Rensselaer Poly technic Institute, Troy, NY, and the S.M. and Ph.D. degrees in mechanical engineering from the Massachusetts Institute of Technology, Cambridge, in 1975 and 1980 , respectively.

He worked as a consultant to the Children's Hospital Medical Center, Boston, MA, during 1979 in bioelectric signal processing, an area in which he is still active. He is currently a Research Scientist in the Applied Dynamics and Acoustics Section of Battelle Columbus Laboratories, Columbus, $\mathrm{OH}$. His research activities there involve the application of both high- and low-intensity ultrasonics in the areas of nondestructive testing of printed-circuit boards, leak detection in nuclear piping systems, and material bonding and unique applications for the oil and gas industry.

Dr. Stulen is a member of the American Society of Mechanical Engineers and Sigma $\mathbf{X i .}$

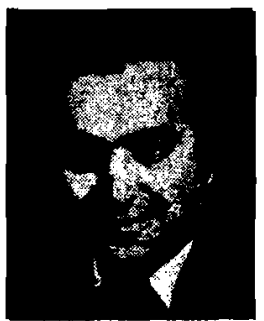

Carlo J. De LuCa (S'64-M'70-SM'77) was borm in Italy in 1943. He received the B.A.Sc. degree in electrical engineering from the University of British Columbia, Vancouver, B.C., Canada, in 1966, the M.Sc. degree in electrical engineering from the University of New Brunswick, Fredericton, N.B., Carada, in 1968, and the Ph.D. degree in biomedical engineering from Queen's Univer sity, Kingston, Ont., Canada, in 1972.

$\mathrm{He}$ is the Director of the Neuromuscular Research Laboratory at Children's Hospital Medical Center, Boston, MA. He currently holds the following appointments: Research Associate in Orthopedic Surgery at Children's Hospital Medical Center, Boston, MA; Principal Research Associate in Orthopedic Surgery (Anatomy) at Harvard Medical School, Cambridge, MA; Adjunct Associate Professor at Boston University, Boston, MA; Project Director, Liberty Mutual Insurance Company Research Center, Hopkinton, MA; Lecturer at Massachusetts Institute of Technology, Cambridge; and Research Member at Harvard-M.I.T. Division of Health Science and Technology. His research interests are in the areas of detection, analysis, and applications of myoelectric and neuroelectric signals directed towards the understanding of motor control. He is the author of approximately 60 scientific publications in these areas. 